\title{
Combined Endoscopic and Oral Fecal Microbiota Transplantation in Patients with Antibiotic- Dependent Pouchitis: Low Clinical Efficacy due to Low Donor Microbial Engraftment
}

\author{
Hans Herfarth ${ }^{a, b}$ Edward L. Barnes ${ }^{a, b}$ Millie D. Long ${ }^{a, b} \quad K^{2}$ L. Isaacs ${ }^{a, b}$ \\ Tom Leith $^{c}$ Michael Silverstein ${ }^{d}$ Ylaine Gerardin ${ }^{d}$ Zain Kassam ${ }^{c, d}$ \\ a Division of Gastroenterology and Hepatology, University of North Carolina, Chapel Hill, NC, USA; \\ b University of North Carolina Multidisciplinary Center for Inflammatory Bowel Diseases, Chapel Hill, NC, USA; \\ ${ }^{c}$ OpenBiome, Somerville, MA, USA; ${ }^{d}$ Finch Therapeutics Group, Somerville, MA, USA
}

\section{Keywords}

Fecal microbiota transplantation · Pouchitis · Inflammatory bowel diseases $\cdot$ Microbiome $\cdot$ Stool transplant

\begin{abstract}
Background and Objective: A significant number of pouch patients develop antibiotic-dependent pouchitis (ADP). Microbial dysbiosis is thought to be a major driver of clinical symptoms in ADP. The objective of this proof of concept study was to evaluate safety, efficacy, and donor microbial engraftment of an intensified fecal microbiota transplant (FMT) consisting of a single endoscopic FMT followed by daily oral FMT for 2 weeks in patients with ADP. Methods: We performed a prospective placebo-controlled doubleblind FMT trial in patents with established ADP and planned to enroll 20 patients in this proof of concept study. In case of non-response, patients were offered an optional open label active FMT treatment. The endpoints were safety, clinical remission without need for antibiotics during 16 weeks of follow-up, quantitative changes of fecal calpro-
\end{abstract}

tectin (FCP), and engraftment of donor FMT as determined by metagenomic sequencing of the $\mathrm{V} 4$ region of the $16 \mathrm{~S}$ rRNA gene. Results: Due to a lower than expected clinical remission rate and low FMT engraftment, enrollment in the study was stopped prematurely after 6 patients were included. All 6 patients enrolled in the placebo-controlled portion failed to respond and needed antibiotic rescue therapy shortly after FMT. FCP increased in the majority of patients in the setting of relapse after FMT. In the active open label FMT extension study 1 out of 5 patients achieved antibiotic-free clinical remission. FMT engraftment after active FMT was observed only in this single patient, whereas engraftment of donor FMT occurred in none of the other patients receiving active FMT, paralleling the lack of clinical response. Conclusions: Low donor FMT engraftment resulted in low clinical efficacy of FMT in patients with ADP. Before embarking on larger clinical trials with FMT in patients with ADP or other forms of pouchitis, it is mandatory to explore approaches for superior FMT engraftment.

(c) 2019 S. Karger AG, Basel

\section{KARGER}

(c) 2019 S. Karger AG, Basel

E-Mail karger@karger.com

www.karger.com/iid
Hans Herfarth, MD, PhD

Department of Medicine, Division of Gastroenterology and Hepatology

University of North Carolina, Bioinformatics Building, CB 7080

Chapel Hill, NC 27599 (USA)

E-Mail hherf@med.unc.edu 


\section{Background}

Due to a refractory course of ulcerative colitis (UC) or histologically proven dysplasia, approximately $20-35 \%$ of patients with UC eventually undergo colectomy, which is most often performed in conjunction with an ileal pouchanal anastomosis (IPAA) [1]. Pouchitis is the most common long-term complication after IPAA, which is clinically defined by the sudden onset of significantly increased bowel frequency and urgency, crampy abdominal pain, and sometimes bloody bowel movements $[2,3]$. Pouchitis can be treated successfully with antibiotics but relapse of pouchitis is common [4]. A significant number of patients, who are in need of recurrent antibiotic therapy to control their pouchitis symptoms, develop antibiotic-dependent pouchitis (ADP). Fecal microbiota transplantation (FMT) is an established therapeutic option for patients with recurrent Clostridioides difficile infection, and is also a promising therapeutic modality for patients with active UC with the aim of re-establishing the homeostasis of the intestinal microbiome [5, 6]. Microbial dysbiosis, which can only be controlled with antibiotics, is thought to be a major driver of clinical symptoms in patients with ADP [7]. A preliminary pilot study recently reported a sustained response for at least 3 months in 3 out of 5 ADP patients after FMT [8]. However, at least 3 endoscopically applied FMTs (eFMTs) were necessary to achieve a response. Repeated eFMTs are costly and impractical. The optimal approach for successful engraftment of donor FMT would be a combination of eFMT (the endoscopy before FMT would also rule out other pouch complications such as strictures) followed by recurring oral encapsulated FMT (oFMT), which only recently became available $[9,10]$. The objective of this single-center, double-blind, randomized placebo-controlled proof of concept study was to evaluate the safety of eFMT followed by daily oFMT to facilitate higher engraftment in patients with ADP. The secondary aims were to evaluate the clinical effectiveness and the engraftment success to estimate the effect size for a subsequent evaluation in a larger definitive trial.

\section{Methods}

Patients with ADP were enrolled in the trial. ADP was defined by the need for continuous antibiotic therapy ( $>4$ weeks) to maintain clinical remission and a history of at least 2 attempts in the last 24 months to stop antibiotic therapy resulting in pouchitis episodes or by active pouchitis with a modified pouch activity index (mPDAI) $\geq 5$ and a history of $\geq 4$ antibiotic therapies for pouchitis in the last 12 months [11]. Clinical remission (mPDAI $<4$ with corresponding
mPDAI clinical subscores for each of the patient-reported outcomes of bowel frequency and urgency of $\leq 1$ ) was induced in all patients with antibiotic therapy, which was stopped at least $24 \mathrm{~h}$ before FMT. A single, rigorously screened stool donor was used from a stool bank whose screening program has been previously described [12]. The stool bank provided frozen eFMT and matching placebo, which contained food colorant to replicate the fecal color, as well as oFMT and matching placebo capsules. All patients meeting the predefined criteria were randomized in a 1:1 ratio using a permuted block design with a fixed block size of 4 to undergo active eFMT $(2 \times 30 \mathrm{~mL}$, total of $24 \mathrm{~g}$ of donor stool) or placebo eFMT followed by daily active oFMT ( $6 \mathrm{G} 3$ capsules gave a total of $4.2 \mathrm{~g}$ of donor stool) or identical placebo capsules for 14 days. Patients who experienced a relapse during the first 4 weeks after endoscopic transplant were offered open label active eFMT followed by 2 weeks of daily active oFMT. The primary endpoint of this study was the safety of the combined eFMT and oFMT approach. The secondary endpoints included remission defined as an mPDAI $<4$ and no need for antibiotics in weeks 4,8 , and 16 after eFMT, quantitative changes of fecal calprotectin (FCP), and engraftment of donor FMT. Stool samples for microbiome and FCP analyses were collected before and at the end of oFMT, and in the case of relapse, before the restart of antibiotics. Metagenomic sequencing of the $\mathrm{V} 4$ region of the $16 \mathrm{~S}$ rRNA gene was performed as previously described, and operational taxonomic units (OTUs) identified as unique sequences present in at least two samples [13]. The counts table was rarefied to the minimum total read count (2,645 reads) and OTU engraftment determined based on presence in the donor sample, depletion in the pre-FMT patient sample, and enrichment in the earliest post-FMT sample.

This trial was designed as a proof-of-concept study of FMT therapy, not a definitive evaluation of FMT therapy in patients with ADP. The goal was to evaluate the safety and feasibility of such a trial, as well as to estimate the effect sizes in placebo and FMT therapy groups in order to accurately estimate the sample size needed for subsequent evaluation in a larger definitive trial. We planned to enroll 20 patents and estimated the minimum effect size this pilot study could detect. We assumed a placebo remission rate (defined as clinical remission and no need for antibiotics) of $10 \%$. A recent case series by Stallmach et al. [8] suggested an antibiotic-free remission rate of $80 \%$ and a response rate of $100 \%$ in the setting of repeated FMT. With 20 patients (1:1 randomization), and a placebo response rate of $10 \%$, we had an $80 \%$ power to detect an effect difference of $67.5 \%$, that is, an antibiotic-free remission rate of $77.5 \%$ in the treatment group (2-sided, alpha $=0.05$, Fisher-Irwin's exact conditional test).

The institutional review board at the University of North Carolina approved the protocol and patients provided written informed consent before entering the trial. A data and safety monitoring board advised the investigators throughout the trial. All authors had access to the study data and reviewed and approved the final manuscript. The trial was registered under ClinicalTrials.gov ID NCT02782325.

\section{Results}

Seven patients were screened and 6 patients were randomly assigned to receive active or placebo FMT (4 patients received active FMT, 2 patients received inactive placebo FMT; Fig. 1; Table 1). All patients experienced 


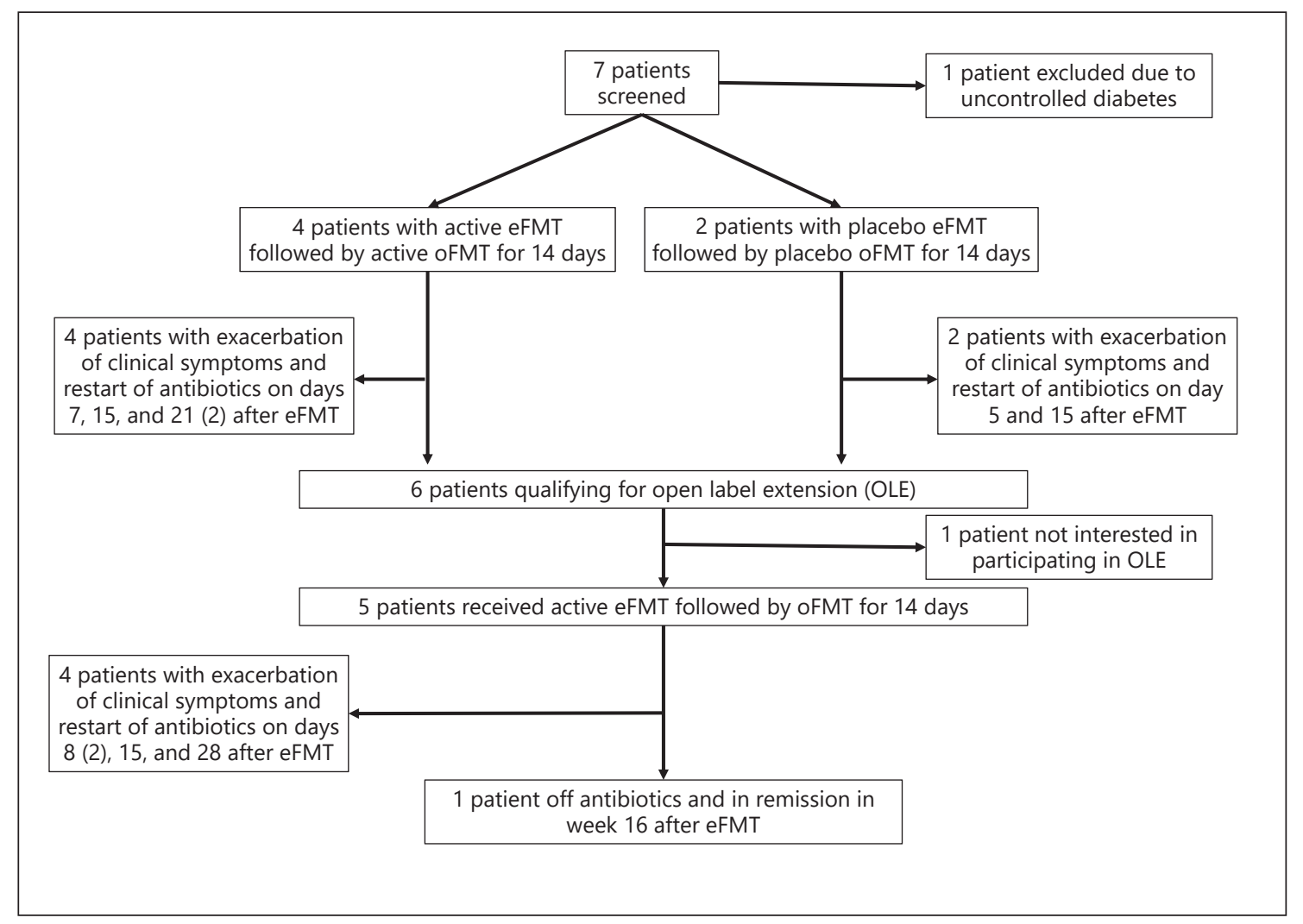

Fig. 1. Consort diagram of the trial.

Table 1. Patient characteristics and mPDAI and FCP before and after FMT

\begin{tabular}{|c|c|c|c|c|c|c|c|c|c|c|c|}
\hline Sex & $\begin{array}{l}\text { Age, } \\
\text { years }\end{array}$ & $\begin{array}{l}\text { Duration, years; } \\
\text { extent of colitis } \\
\text { before colectomy }\end{array}$ & $\begin{array}{l}\text { Indication for } \\
\text { colectomy }\end{array}$ & $\begin{array}{l}\text { IPAA } \\
\text { stage }\end{array}$ & $\begin{array}{l}\text { Duration } \\
\text { postop. until } \\
\text { first pouchitis, } \\
\text { months }\end{array}$ & $\begin{array}{l}\text { Time since } \\
\text { IPAA before } \\
\text { inclusion } \\
\text { in trial, years }\end{array}$ & $\begin{array}{l}\text { Antibiotic therapy } \\
\text { for ADP }\end{array}$ & $\begin{array}{l}\text { FMT } \\
\text { transplant } \\
\text { sequence }\end{array}$ & $\begin{array}{l}\text { mPDAI at inclusion } \\
\left(\text { clinical symptoms }{ }^{2}\right) \\
\text { pouchoscopies, } n\end{array}$ & $\begin{array}{l}\text { mPDAI (clinical } \\
\text { symptoms }{ }^{2} \text { ) before } \\
\text { restart anti-biotics: } \\
\text { rFMT; olFMT }\end{array}$ & $\begin{array}{l}\text { FCP screening; before } \\
\text { restart antibiotics in } \\
\text { rFMT; olFMT, } \mu \mathrm{g} / \mathrm{g} \\
\text { stool }\end{array}$ \\
\hline M & 60 & $\begin{array}{l}\text { 11; left-sided } \\
\text { colitis }\end{array}$ & Other ${ }^{1}$ & 3 & 24 & 10 & Ciprofloxacin & Ac, Ac & $0 ; 2$ & $2 ; 2$ & $101 ; 150 ; 224$ \\
\hline $\mathrm{F}$ & 35 & 8 ; proctitis & Refractory & 1 & 1 & 3 & Ciprofloxacin & $\mathrm{Pl}, \mathrm{Ac}$ & $1 ; 1$ & $2 ; 1^{3}$ & $321 ; 180 ; 55$ \\
\hline M & 34 & 15; pancolitis & $\begin{array}{l}\text { Refractory + } \\
\text { dysplasia }\end{array}$ & 2 & 1 & 5 & Amoxicillin & Ac, n.p. & $1 ; 0$ & $2 ;$ n.p. & $65 ; 720 ; n p$ \\
\hline $\mathrm{F}$ & 32 & $\begin{array}{l}\text { 2; left-sided } \\
\text { colitis }\end{array}$ & Refractory & 2 & 14 & 10 & Ciprofloxacin & $\mathrm{Pl}, \mathrm{Ac}$ & $0 ; 0$ & $2 ; 2$ & 69; n.d.; 18 \\
\hline $\mathrm{M}$ & 22 & 1; pancolitis & Refractory & 2 & 1 & 1 & Ciprofloxacin & Ac, Ac & $1 ; 1$ & $3 ; 4$ & $21 ; 254 ; 226$ \\
\hline M & 41 & 1 ; pancolitis & Refractory & 3 & 12 & 5 & Metronidazole & Ac, Ac & $1 ; 0$ & $3 ; 4$ & $210 ; 399 ; 454$ \\
\hline
\end{tabular}

mPDAI, modified pouch activity index; FCP, fecal calprotectin; FMT, fecal microbiota transplant; rFMT, FMT in randomized phase; olFMT, FMT in open label phase; n.p., not performed since patient did not participate in open label extension; n.d., patient did not collect stool sample; Pl, placebo FMT; Ac, active FMT.

${ }^{1}$ Sigmoid perforation.

2 The clinical symptoms patients reported for stool frequency and urgency were evaluated daily for 3 days, with a minimum combined score of 0 and a maximum score of 4 . Stool frequency: score 0 , usual postop. stool frequency in the last 3 days; score $1,1-2$ stools/day > postop. usual; score 2 , 3 or more stools/day > post-op. usual. Urgency: score 0 , none, $0 \%$ of bowel movements; score 1 , occasional, $<50 \%$ of bowel movements; score 2 , usual, $>50 \%$ of bowel movements.

${ }^{3}$ Patient did not restart antibiotics and remained in remission with a clinical PDAI of 1 throughout the trial period. 


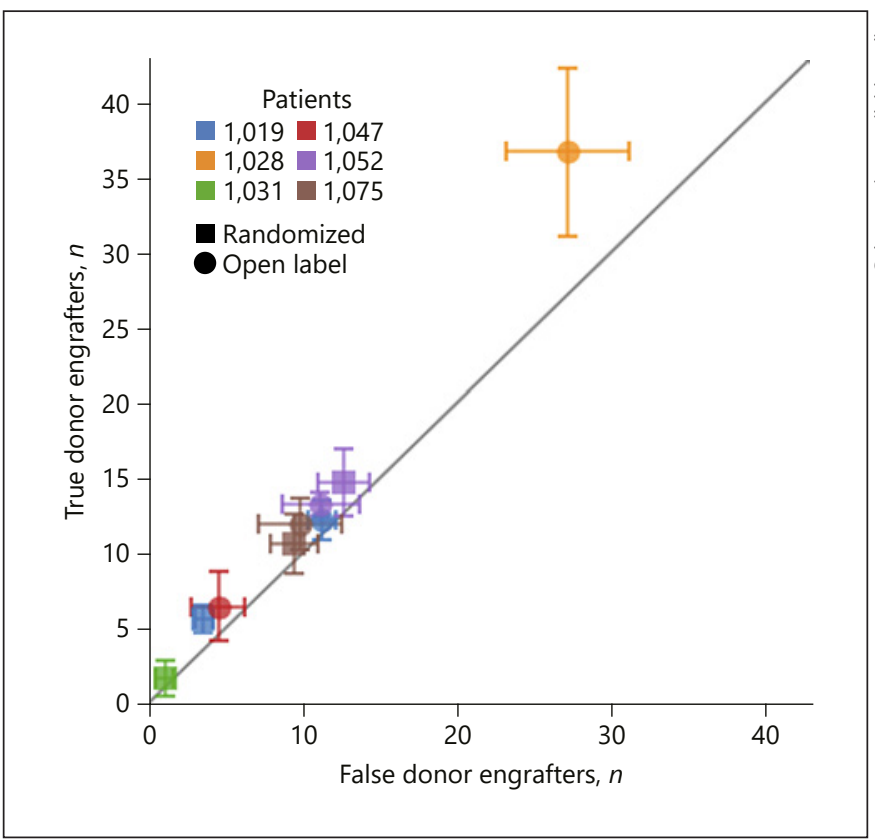

Fig. 2. eFMT followed by oFMT in 6 patients. In total, 9 active FMTs were performed in the 6 patients; 3 patients were treated with active FMT in the blinded randomized as well as in the open label part of the study, 1 patient was treated with active FMT in the randomized part only, and 2 patients in the open label part only. The chart shows the number of OTUs that were engrafted from samples of a patient's true donor compared to the number of OTUs that were engrafted from a set of 10 "false donors," which were randomly selected from the stool bank donor pool (error bars show standard error). Only 1 patient (patient 1,028, who first received placebo FMT and then active FMT in the open label extension) showed strong engraftment specificity, as shown by the significantly higher number of engrafting OTUs inferred from the true donor versus the false donors. This patient also experienced stable clinical symptoms after FMT and did not restart antibiotics during the trial period of 16 weeks.

relapse with increase of diarrhea and urgency either during or shortly after the completion of FMT. After the reinduction of remission with antibiotic therapy, 5 patients continued with open label active FMT (Fig. 1). All but 1 patient experienced a relapse with increase of clinical symptoms during or shortly after the completion of FMT therapy. Overall, FMT was well tolerated and no FMTrelated safety events were observed. Due to the unexpected low efficacy of FMT in the open label segment of the study, the data and safety committee together with the principal investigator of the study decided to halt enrollment and perform an interim analysis to evaluate FMT engraftment and FCP values. FCP increased in nearly all patients with relapse (Table 1). In most patients the Shan- non alpha diversity was enhanced after the concurrent stop of antibiotics and FMT (results not shown), but FMT-specific engraftment only occurred in the 1 patient who clinically responded to FMT and remained off antibiotics for the study period of 16 weeks (Fig. 2). However, this patient had to restart antibiotic therapy approximately 3 weeks after conclusion of the trial due to worsening diarrhea.

\section{Discussion}

This proof of concept study of intensified FMT combining endoscopic FMT with a daily oral maintenance FMT did not show a significant clinical effect in patients with ADP. Only 1 out of 6 patients (17\%) receiving active FMT was able to stay off antibiotics for a longer time period after FMT. Microbiome analyses revealed that only this patient successfully engrafted donor FMT, whereas all other patients receiving active FMT showed no engraftment.

To our knowledge, this is the first study evaluating the engraftment of donor FMT in patients with an ileal pouch. Despite the small sample size, the results suggest that donor FMT engraftment in this patient group is surprisingly low despite a combined endoscopic and oral high-intensity FMT approach, which is considered one of the factors of success for FMT in patients with UC [14]. In contrast, engraftment of FMT in patients with a preserved colon in FMT trials for recurrent $C$. difficile infection and UC is at least $80 \%[15,16]$. Also, as shown in patients with UC undergoing FMT, the microbiome of recipients after transplant is more similar to the donor FMP than the pretransplant recipient sample engraftment, suggesting that FMT engraftment is a prerequisite for therapeutic success [16]. The reason for the low engraftment in our study could be due to several factors, including donor selection, the dose and frequency of FMT, and the pouch microenvironment. The low donor engraftment found in our study may also explain the inconsistent and partially contradictory results of the other small controlled and uncontrolled observations of FMT in patients with chronic refractory pouchitis or ADP $[8$, 17]. Further studies need to determine the mechanisms of engraftment in the small bowel environment. Furthermore, analyses which discern the FMT donor engraftment after endoscopic versus oral FMT may reveal the optimal dosing and route of FMT application in this patient group. The microbial diversity increased in placebo as well as in active FMT patients most likely due to the 
stop of the antibiotics before FMT. Antibiotics decrease microbial diversity, which is re-established after FMT in parallel with successful FMT engraftment [18]. However, the increase of microbial diversity in the study patients was overall low, which corresponded to the low donor FMT engraftment.

Increased bowel frequency and significant urgency were the main signs of a lack of response to FMT in all patients. These symptoms improved $24 \mathrm{~h}$ after the re-initiation of antibiotic therapy. FCP levels above $50 \mu \mathrm{g} / \mathrm{g}$ of stool have been associated with pouchitis, but validation studies in this patient group are not available [19]. Also in patients with UC, levels between 200 and $250 \mu \mathrm{g}$ have been associated with significant histologic inflammation [20]. At inclusion in our study, FCP levels were between $>50$ and $<250 \mu \mathrm{g} / \mathrm{g}$ of stool, respectively, in 5 out of 6 (83\%) of the patients. After the patients reported increased symptoms before the re-initiation of antibiotics, FCP levels had increased to $>250 \mu \mathrm{g} / \mathrm{mg}$ in $50 \%$ of the patients. The FCP level in the only patient who responded to FMT was below $50 \mu \mathrm{g} / \mathrm{g}$ of stool at the end of 16 weeks of follow-up.

We based our power calculations on the previous observations by Stallmach et al. [8] in 5 patients with refractory ADP reporting a response rate of $100 \%$ and a remission rate of $80 \%$. Since our targeted patient group was antibiotic dependent but not antibiotic refractory, we conservatively estimated a remission rate of $80 \%$ and assumed a placebo response of $10 \%$. After only 1 out of 6 (17\%) patients was in remission and off antibiotics after active FMT, the data safety monitoring board together with the principal investigator decided to stop the study early due to the unexpected low remission rate.

The low remission rate and low engraftment may be due to the fact that the transplantation was performed with a single donor and not with a multidonor approach, as described in a recent FMT study in patients with UC [21]. The Food and Drug Agency (FDA) investigational new drug application, which is necessary for every clinical study in the USA and authorizes the administration of an investigational drug or biological product to humans, did not allow the use of a multidonor approach for the endoscopic and capsulated FMT in our study. Thus, we tried to optimize the single-donor approach by choosing a relatively high-butyrate producer (approx. 1.4 deviations above other donors). Higher butyrate levels have been associated with a higher microbial diversity and better therapeutic response rates in previous FMT trials in patients with recurrent clostridium difficile infection, UC, or metabolic syndrome [22-25].
In conclusion, intensified FMT in patients with ADP, who appear the optimal candidates for FMT therapy due to the suspected microbial dysbiosis as the underlying pathomechanism of their symptoms, was associated with a remarkably low engraftment and therapeutic success. Thus, before embarking on larger clinical studies with FMT in patients with ADP or other forms of pouchitis, it is mandatory to explore approaches for superior FMT engraftment in this patient group.

\section{Statement of Ethics}

The institutional review board at the University of North Carolina approved the protocol and patients provided written informed consent before entering the trial. A data and safety monitoring board advised the investigators throughout the trial. All authors had access to the study data and reviewed and approved the final manuscript. The trial was registered under ClinicalTrials.gov ID NCT02782325.

\section{Disclosure Statement}

H.H. has received consulting fees from Alivio, AMAG, Merck, Pfizer, Celltrion, Lycera, Boehringer-Ingelheim, and Seres, research support from Pfizer and Artizan, and is member of a data and safety monitoring board at Finch and Gilead. E.L.B. has received consulting fees from Janssen Biotech Inc. M.D.L. has received consulting fees from Abbvie, Takeda, Pfizer, and Theravance, and research support from Pfizer and Takeda. All other authors report no disclosures or conflicts of interest relevant to this study.

\section{Funding Sources}

The study was funded by Litwin IBD Pioneers initiative at the Crohn's and Colitis Foundation and the National Institutes of Health (P30 DK034987).

\section{Author Contributions}

H.H., Z.K.: conception and design of study. H.H., E.L.B., M.D.L., K.L.I., T.L., M.S., Y.G., and Z.K.: acquisition and analysis of data, critical revision of the report. All authors approved the final submission. 


\section{References}

1 Bernstein CN, Ng SC, Lakatos PL, Moum B, Loftus EV Jr; Epidemiology and Natural History Task Force of the International Organization of the Study of Inflammatory Bowel Disease. A review of mortality and surgery in ulcerative colitis: milestones of the seriousness of the disease. Inflamm Bowel Dis. 2013 Aug;19(9):2001-10.

2 Barnes EL, Herfarth HH, Sandler RS, Chen W, Jaeger E, Nguyen VM, et al. Pouch-Related Symptoms and Quality of Life in Patients with Ileal Pouch-Anal Anastomosis. Inflamm Bowel Dis. 2017 Jul;23(7):1218-24.

3 Shen B, Fazio VW, Remzi FH, Lashner BA. Clinical approach to diseases of ileal pouchanal anastomosis. Am J Gastroenterol. 2005 Dec;100(12):2796-807.

4 Segal JP, Ding NS, Worley G, Mclaughlin S, Preston S, Faiz OD, et al. Systematic review with meta-analysis: the management of chronic refractory pouchitis with an evidence-based treatment algorithm. Aliment Pharmacol Ther. 2017 Mar;45(5):581-92.

5 Kassam Z, Lee CH, Yuan Y, Hunt RH. Fecal microbiota transplantation for Clostridium difficile infection: systematic review and meta-analysis. Am J Gastroenterol. 2013 Apr; 108(4):500-8.

6 Allegretti J, Eysenbach LM, El-Nachef N, Fischer M, Kelly C, Kassam Z. The Current Landscape and Lessons from Fecal Microbiota Transplantation for Inflammatory Bowel Disease: Past, Present, and Future. Inflamm Bowel Dis. 2017 Oct;23(10):1710-7.

7 Machiels K, Sabino J, Vandermosten L, Joossens M, Arijs I, de Bruyn M, et al. Specific members of the predominant gut microbiota predict pouchitis following colectomy and IPAA in UC. Gut. 2017 Jan;66(1):79-88.

8 Stallmach A, Lange K, Buening J, Sina C, Vital M, Pieper DH. Fecal Microbiota Transfer in Patients With Chronic Antibiotic-Refractory Pouchitis. Am J Gastroenterol. 2016 Mar; 111(3):441-3.

9 Stollman N, Smith M, Giovanelli A, Mendolia G, Burns L, Didyk E, et al. Frozen encapsulated stool in recurrent Clostridium difficile: exploring the role of pills in the treatment hierarchy of fecal microbiota transplant nonresponders. Am J Gastroenterol. 2015 Apr; 110(4):600-1.
10 Fischer M, Allegretti JR, Smith M, Klank MJ, Mendiola G, Vo E, et al. A multi-center, cluster randomized dose-finding study of fecal microbiota transplantation capsules for recurrent clostridium difficile infection. United Eur Gastroenterol J. 2015;3:565.

11 Shen B, Achkar JP, Connor JT, Ormsby AH, Remzi FH, Bevins CL, et al. Modified pouchitis disease activity index: a simplified approach to the diagnosis of pouchitis. Dis Colon Rectum. 2003 Jun;46(6):748-53.

12 Burns LJ, Dubois N, Smith MB, Mendolia GM, Burgess J, Edelstein C, et al. 499 donor recruitment and eligibility for fecal microbiota transplantation: results from an international public stool bank. Gastroenterology. 2015;148(4):S96-7.

13 Kozich JJ, Westcott SL, Baxter NT, Highlander SK, Schloss PD. Development of a dual-index sequencing strategy and curation pipeline for analyzing amplicon sequence data on the MiSeq Illumina sequencing platform. Appl Environ Microbiol. 2013 Sep;79(17):5112-20.

14 Siegmund B. Is intensity the solution for FMT in ulcerative colitis? Lancet. 2017 Mar;389 (10075):1170-2.

15 Smillie CS, Sauk J, Gevers D, Friedman J, Sung J, Youngster I, Hohmann EL, Staley C, Khoruts A, Sadowsky MJ, Allegretti JR, Smith MB, Xavier RJ, Alm EJ. Strain tracking reveals the determinants of bacterial engraftment in the human gut following fecal microbiota transplantation. Cell Host Microbe. 2018;23:229-240.e5.

16 Jacob V, Crawford C, Cohen-Mekelburg S, Viladomiu M, Putzel GG, Schneider Y, et al. Single Delivery of High-Diversity Fecal Microbiota Preparation by Colonoscopy Is Safe and Effective in Increasing Microbial Diversity in Active Ulcerative Colitis. Inflamm Bowel Dis. 2017 Jun;23(6):903-11.

17 Landy J, Walker AW, Li JV, Al-Hassi HO, Ronde E, English NR, et al. Variable alterations of the microbiota, without metabolic or immunological change, following faecal microbiota transplantation in patients with chronic pouchitis. Sci Rep. 2015 Aug;5(1): 12955.
18 Taur Y, Coyte K, Schluter J, Robilotti E, Figueroa C, Gjonbalaj M, et al. Reconstitution of the gut microbiota of antibiotic-treated patients by autologous fecal microbiota transplant. Sci Transl Med. 2018 Sep;10(460):10.

19 Yamamoto T, Shimoyama T, Bamba T, Matsumoto K. Consecutive Monitoring of Fecal Calprotectin and Lactoferrin for the Early Diagnosis and Prediction of Pouchitis after Restorative Proctocolectomy for Ulcerative Colitis. Am J Gastroenterol. 2015 Jun;110(6): 881-7.

20 Magro F, Lopes J, Borralho P, Lopes S, Coelho $\mathrm{R}$, Cotter J, et al. Comparison of different histological indexes in the assessment of UC activity and their accuracy regarding endoscopic outcomes and faecal calprotectin levels. Gut. 2019;68(4):594-603.

21 Paramsothy S, Kamm MA, Kaakoush NO, Walsh AJ, van den Bogaerde J, Samuel D, et al. Multidonor intensive faecal microbiota transplantation for active ulcerative colitis: a randomised placebo-controlled trial. Lancet. 2017 Mar;389(10075):1218-28.

22 Fuentes S, Rossen NG, van der Spek MJ, Hartman JH, Huuskonen L, Korpela K, et al. Microbial shifts and signatures of long-term remission in ulcerative colitis after faecal microbiota transplantation. ISME J. 2017 Aug; 11(8):1877-89.

23 Vrieze A, Van Nood E, Holleman F, Salojarvi J, Kootte RS, Bartelsman JF, et al. Transfer of intestinal microbiota from lean donors increases insulin sensitivity in individuals with metabolic syndrome. Gastroenterology. 2012; 143:913-6.e7.

24 Kelly CR, Kahn S, Kashyap P, Laine L, Rubin $\mathrm{D}$, Atreja A, et al. Update on fecal microbiota transplantation 2015: indications, methodologies, mechanisms, and outlook. Gastroenterology. 2015 Jul;149(1):223-37.

25 Barnes D, Ng K, Smits S, Sonnenburg J, Kassam Z, Park KT. Competitively Selected Donor Fecal Microbiota Transplantation: Butyrate Concentration and Diversity as Measures of Donor Quality. J Pediatr Gastroenterol Nutr. 2018 Aug;67(2):185-7. 